\title{
Factors causing oral and skin pathological features in the hyperimmunoglobulin E syndrome patient including the environmental component: a review of the literature and own experience
}

\author{
Karolina Gerreth ${ }^{1}$, Aleksandra Szczawinska-Poplonyk², Zdzislawa Kycler², Zygmunt Adamski ${ }^{3}$ \\ Maria Borysewicz-Lewicka ${ }^{4}$, Anna Breborowicz ${ }^{2}$
}

\begin{abstract}
${ }^{1}$ Department of Risk Group Dentistry, Chair of Paediatric Dentistry, Poznan University of Medical Sciences, Poznan, Poland ${ }^{2}$ Department of Paediatric Pneumonology, Allergology and Clinical Immunology, $3^{\text {rd }}$ Chair of Paediatrics, Poznan University of Medical Sciences, Poznan, Poland

${ }^{3}$ Department of Dermatology, Poznan University of Medical Sciences, Poznan, Poland

${ }^{4}$ Chair and Department of Paediatric Dentistry, Poznan University of Medical Sciences, Poznan, Poland
\end{abstract}

Adv Dermatol Allergol 2020; XXXVII (3): 326-332

DOI: https://doi.org/10.5114/ada.2020.96142

\begin{abstract}
The hyperimmunoglobulin E syndrome (HIES) is a rare multi-system disease with non-immunological as well as immunological abnormalities. The syndrome is characterized by a triad of the most distinctive symptoms, such as pneumonia with pneumatocele formation, recurring staphylococcal skin abscesses and a high serum concentration of IgE. Central mediators of immune responses such as STAT1 and STAT3 affect immune responses and contribute to changes of the skin microbiome which subsequently can amplify the defective immune response against microbial and fungal pathogens. Reactions related to an environmental factor, such as sun-induced skin changes, in individuals during long-term medication therapy have also been reported. The dermatological symptoms, oral status and other health problems of a hyperimmunoglobulin E syndrome paediatric patient are presented. HIES is of great importance to different professionals because sufferers require special preventive and therapeutic management from early infancy in order to avoid complications which can even prove to be life-saving for such patients.
\end{abstract}

Key words: hyperimmunoglobulin E syndrome (hyper-IgE syndrome, HIES), environmental factor, dermatological manifestation, oral finding.

\section{Introduction}

The hyperimmunoglobulin E syndrome (HIES) is a rare disease with the incidence estimated to be one in 500,000 to $1,000,000$ individuals [1, 2]. The disorder has no sex predilection and it may occur in individuals with diverse ethnic backgrounds [3, 4].

Davis et al. described the syndrome for the first time in 1966, and named the condition as Job's syndrome [5, 6], whereas Buckley et al. reported two other cases with similar manifestations in 1972, and referred to this disorder as Buckley's syndrome [6, 7]. HIES is a multi-system disorder, with non-immunological and immunological abnormalities, and affects the dentition, skeleton, connective tissue and immune system [6, 8-15]. However, the syndrome is characterized by a triad of the most typical symptoms, such as pneumonia with pneumatocele for- mation, recurring staphylococcal skin abscesses as well as a high serum concentration of IgE $[8,10,16]$.

Intraoral manifestations include lesions within the gingival and oral mucosa which involve the lip and buccal mucosa, a hard palate as well as a dorsal tongue [17]. Other oral features comprise a high and arched palate, a failure to shed primary dentition, microdontia as well as supernumerary teeth [1].

Diagnosis of HIES might be delayed up to late childhood or even adulthood despite the fact that the disease often commences very early on in life [4]. Thus, an immediate diagnosis could prevent complications, e.g. pneumatocele formation by the insertion of prophylactic antistaphylococcal therapy [10]. In the syndrome, the immunoglobulin E level is elevated in the blood, therefore, IgE directed against Staphylococcus aureus has a relatively specific diagnostic value [4]. 
It must be emphasized that oral findings manifest earlier than the development of typical facial changes, hence, the expected role of oral phenotypes in early diagnosis of the disorder exists [18].

Two forms of HIES have been described in the literature, i.e. autosomal dominant type of the syndrome (ADHIES) and autosomal recessive hyperimmunoglobulin $E$ syndrome (AR-HIES) [10].

AD-HIES is a result of hypomorphic mutations in the signal transducer and the activator of the transcription 3 (STAT3) gene. Susceptibility to infections in such patients results from a STAT3 mutation which causes a defective multiple cytokine signal transduction, such as IL-6 and IL-22, and the impaired function of Th17 [19]. However, despite the current evidence to suggest that STAT3 is the only gene identified as yet to cause AD-HIES, it is likely that more than one genetic etiologic factor exist [18]. At present, it has been suggested that cytokines link the dental and other immunologic and skeletal manifestations of the disease [1].

AR-HIES seems to have a distinct disorder entity because of a dissimilar infection profile from AD-HIES, frequent malignancy, neurological complications and autoimmunity, even if AD- and AR-HIES share common findings such as susceptibility to cutaneous lesions, staphylococcal infections and hyperimmunoglobulinemia E [19].

HIES sufferers are generally maintained on antifungal and antibacterial medications, with an aggressive therapy of infections as they arise [13]. Thus, the side effects of medicinal interventions are very often detected in the patients, apart from other oral and facial manifestations typical for the disease itself.

\section{Review of literature and own experience}

A 12-year-old girl with HIES was referred to the department of paediatric dentistry for dental diagnosis and treatment. From early infancy the patient had experienced skin abscesses and severe generalized eczema. She had also suffered from recurrent gastrointestinal and respiratory tract infections. At the age of 2 years the girl had pathological fractures of clavicle and both shin-bones. Furthermore, at the age of 3 years she required surgical therapy of an abscess in the right popliteal space. The girl was referred to the university department of paediatric pneumonology, allergology and clinical immunology where she was diagnosed with HIES at the age of 5 years and she has been systematically followed up since.

Over the course of the following years the girl suffered from frequent pneumonia and at the age of 7 years, a lobectomy of the upper part of the right lung was done because of aspergilloma. At the age of 12 years, Scedosporium in patient's lungs was found. Every second year, bronchoscopies to clear the airways and computer tomography for the purposes of monitoring chronic interstitial and parenchymal lung disease were necessary.
Furthermore, the patient was diagnosed with autoimmune polyglandular syndrome with thyroid, adrenal and somatotropic pituitary insufficiency.

An extraoral examination showed deep-set eyes, a prominent forehead and eyebrow, a broadened nasal bridge as well as a fleshy nasal tip. The girl's facial skin exhibited prominent pores and appeared typically coarse. On the left side of the girl's lower lip, mucosal discoloration, brown in colour, was observed which appeared after treatment with the antifungal agent voriconazole (Vfend) (Figure 1).

An intraoral check-up revealed midline sagittal fibrotic thickening covering the anterior part of the hard palate (around half of the entire hard palate) and a high arched palate (Figure 2).

Personal history revealed that the girl's first dental visit was in the $3^{\text {rd }}$ year of life. Her deciduous teeth erupted on time. When the patient was 5 years old she suffered from an abscess next to the upper milk lateral and central incisors, however, as her mother reported, the teeth were only opened after the abscess had been punctured. Moreover, 2 months later she was admitted to the hospital due to an abscess in the right suborbital area. The upper right incisors and canine were extracted under general anaesthesia, and the abscess was surgically incised and drained. During hospital stay the girl was also diagnosed with sinusitis. Additionally, a few months later the patient experienced abscesses four times consecutively from her deciduous teeth and those teeth were extracted.

Retrospective analysis of the girl's dental chart revealed that the patient had first visited the department of paediatric dentistry at the age of 5 years, due to complications of dental caries in the maxillary left primary first molar. At the same age also an orthodontic visit was carried out. A few months later, the lower and upper removable space maintainers were done. The patient has been under constant orthodontic supervision. However,

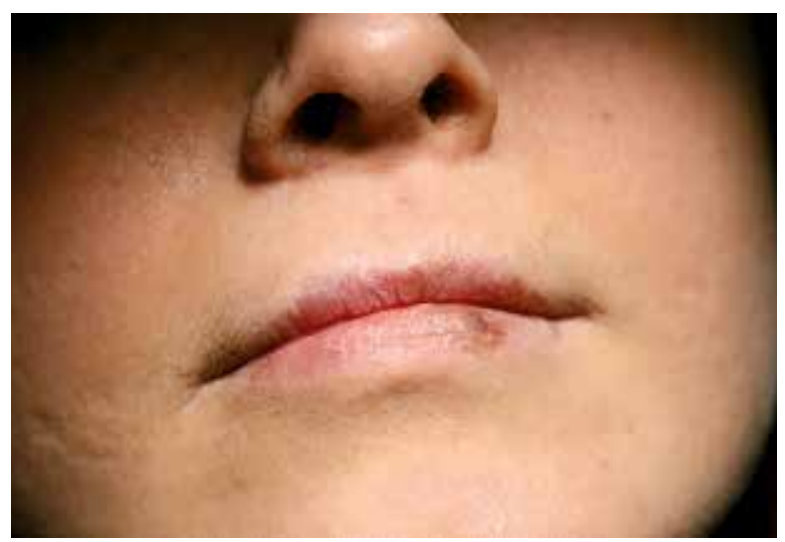

Figure 1. Discoloration of the lower lip reflecting a phototoxic reaction occurring during Voriconazole treatment as well as visible prominent pores on the facial skin 


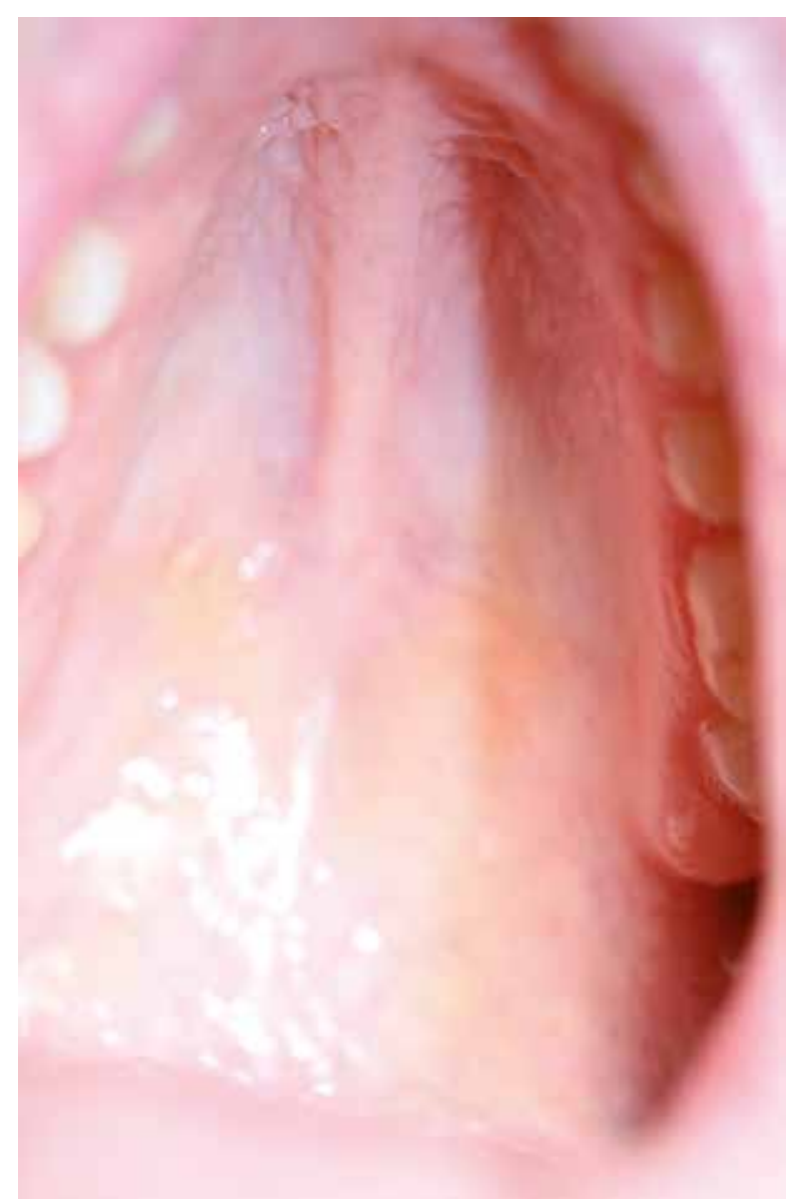

Figure 2. A high arched palate as well as midline sagittal fibrotic thickening covering an anterior part of the hard palate

at the age of 6 years, the girl wore the only lower one due to the eruption of the upper permanent lateral right incisor. She did not use the prosthesis during her numerous stays at hospital, and at the age of 9 years, the treatment was abandoned because of recurrent aphthous ulcers as well as candidiasis within her oral mucosa. Moreover, the patient's mother reported frequent aphthous ulcers since birth.

Oral hygiene instruction, preventive teeth varnishing as well as dental treatment has been done regularly in the patient since she was 7 years old. Moreover, orthodontic indications required the extraction of four deciduous teeth. Because the permanent successors were erupting, and 2 deciduous teeth were still present within the oral cavity, one milk tooth had to be extracted when the girl was 12 years old and the other at the age of 13 . Furthermore, the extractions of 3 deciduous teeth with complications of dental caries such as chronic periodontitis were carried out at the age of 12 years. Conservative treatment was done in 7 permanent teeth with carious lesions. At the age of 14 years, the girl was referred by her orthodontist for surgical exposure of the permanent mandibular left canine and the permanent mandibular right second premolar since there was no eruption. Fortunately, she does not have prognathia which is typical for patients with HIES.

The presence of the persistent deciduous mandibular central right incisor was observed with no germ of a successor since orthopantomograms confirmed that the tooth bud of the permanent right central incisor was absent. Her mother reported that the patient did not experience problems with the eruption of the primary teeth, however, a delayed eruption of permanent teeth was observed. Orthopantomograms were taken regularly to monitor teeth eruption (Figures 3 and 4).

Currently, the girl is 17 years old and remains under the constant supervision of the department of paediatric dentistry and department of pneumonology, allergology and clinical immunology.

The hyperimmunoglobulin E syndrome is a complex immune deficiency that has diverse clinical features because of heterogeneous genetic origins [19].

In AR-HIES dental and skeletal manifestations are absent. There is increased susceptibility to severe viral and fungal infections as well as a high incidence of vascular and infectious central nervous system complications [20].

On the other hand, AD-HIES is characterized by such abnormalities as elevated serum immunoglobulin E levels; lung staphylococcal infections with a predisposition to formation of pneumatoceles; scoliosis and joint hyperextensibility; characteristic faces with facial asymmetry and prominent forehead, deep-set eyes, broad nasal bridge and mild prognathism; decreased bone density leading to a high risk of bone fracture after minimal trauma as well as severe dermatological problems including chronic eczematoid dermatitis and recurrent skin abscesses that often lack the usual signs of inflammation such as warmth, erythema, and tenderness ("cold abscesses") [20].

Such typical clinical features of AD-HIES as furunculosis, eczema, and recurrent pneumonias with lung abscesses and pneumatoceles often become noticeable in infancy or early childhood. It is interesting that our patient has experienced distinguishing skin and lung problems since infancy as her mother reported.

In almost all sufferers, the skin rash usually arises as papules or pustules on the face as well as the scalp in the new-born period [18]. Subsequently the lesions develop over the rest of the body and predominantly mimics eczema. However, it is generally caused by the Staphylococcus aureus infection. The rash is managed with anti-staphylococcal antibiotics or topical treatment such as swimming in chlorinated pools or bathing in diluted bleach [18].

The results of Smeekens et al. study, carried out in chronic mucocutaneous candidiasis (CMC) and hyper IgE syndrome (HIES) patients as well as in healthy controls, revealed that central mediators of immune responses such as 


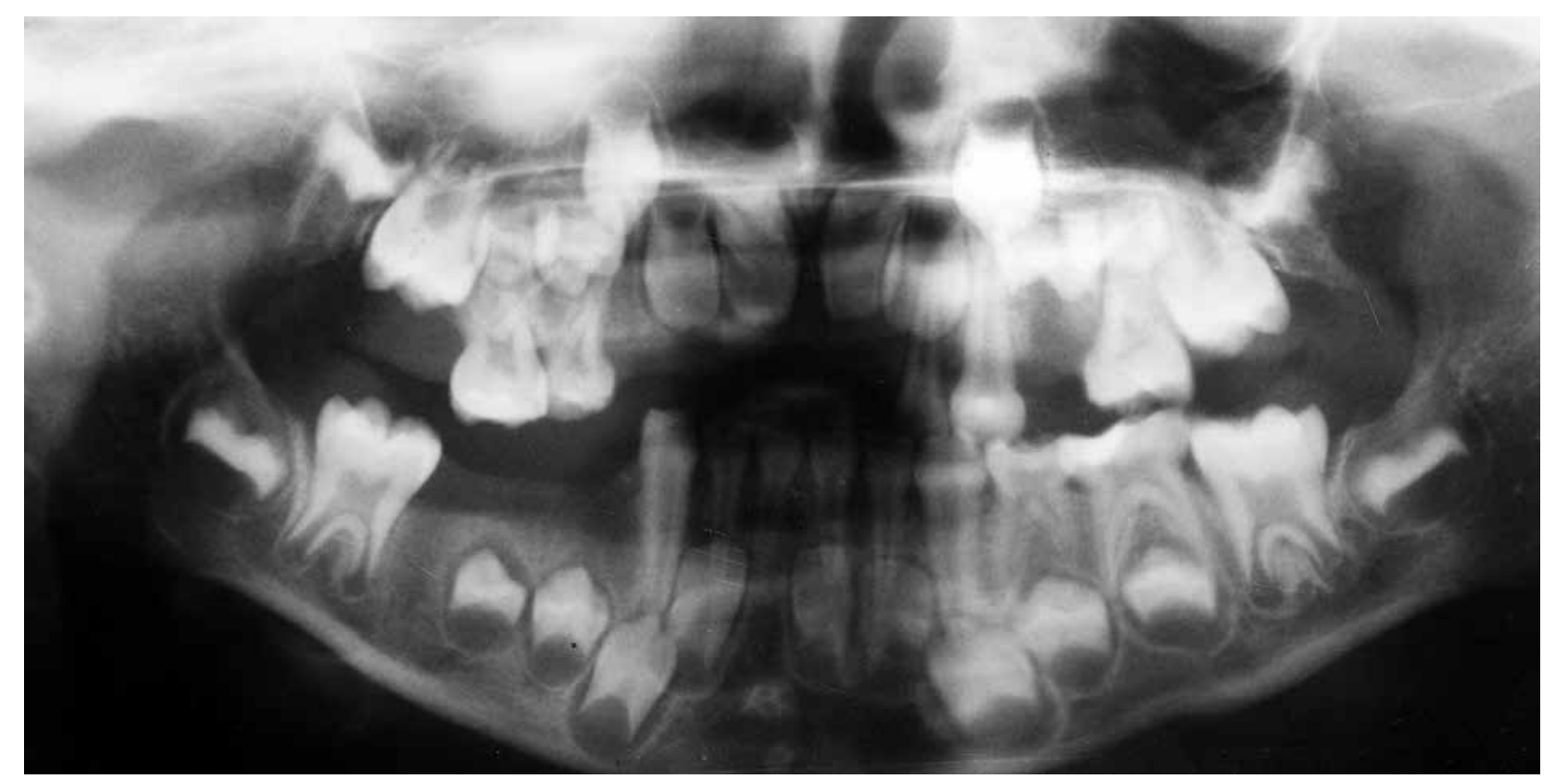

Figure 3. Orthopantomograms taken when the girl was 5 years old

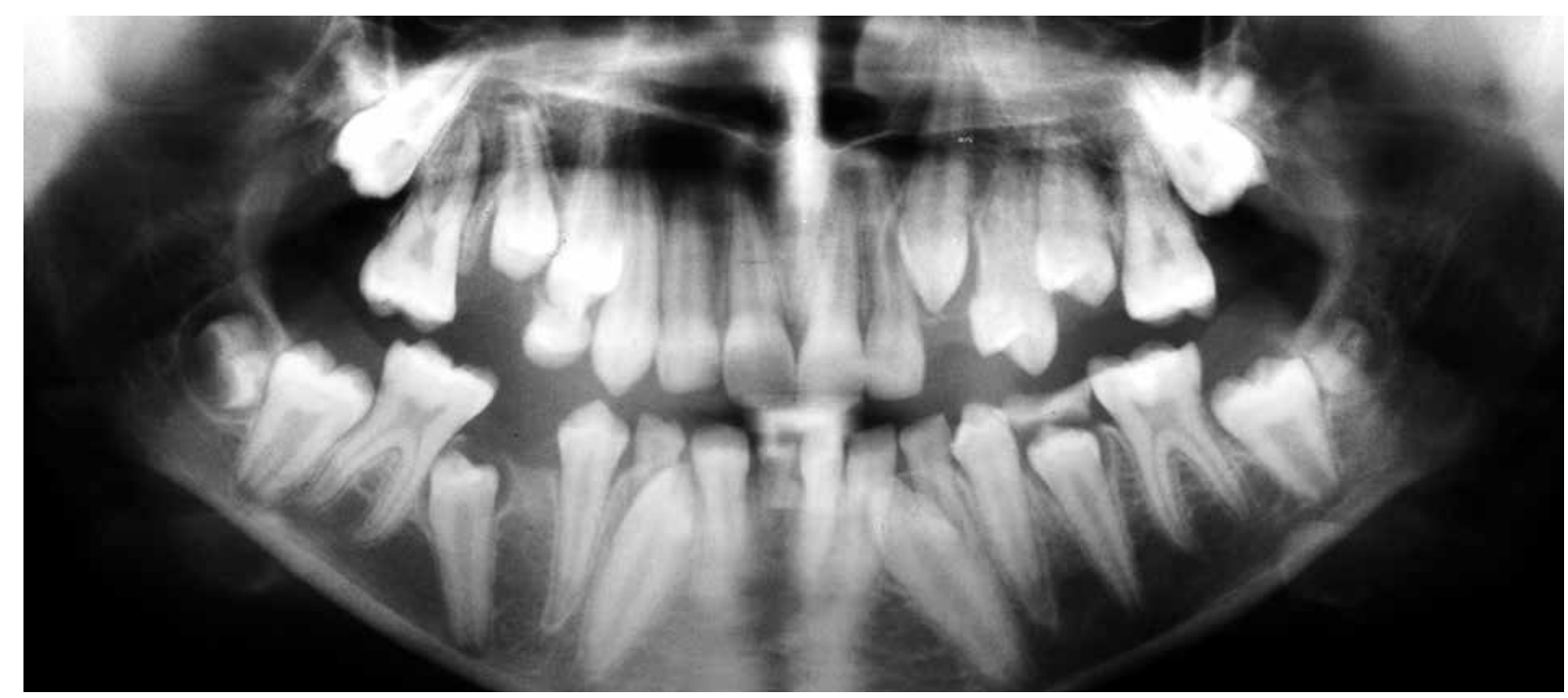

Figure 4. Orthopantomograms taken when the girl was 12 years old

STAT1 and STAT3 not only directly affect immune responses, but also contribute to changes of the skin microbiome that subsequently can amplify the defective immune response against microbial and fungal pathogens [21].

The other symptoms, particularly the typical appearance of a coarse face, may not manifest themselves before adolescence or late childhood [6]. A distinctive coarse face encompasses a prominent brow and a supraorbital ridge with the impression of deep-set eyes, along with a greater interalar width as well as a longer outer canthal distance [4]. Grimbacher et al. revealed characteristic faces in 25 out of 30 patients examined whereas Tonsi observed that $36.67 \%$ of individuals had coarse faces with a prominent brow and deep-set eyes $[3,22]$. Furthermore, Pherwani and Madnani showed dysmorphic facial manifestations in four children out of 6 patients with HIES while prognathia was seen in only one child [23]. Our patient had characteristic facial features of the syndrome such as a prominent forehead and brow, a broadened nasal bridge as well as deep-set eyes. Additionally, a high arched palate was diagnosed, whereas, prognathia was not observed.

The mother of our patient reported that there was also a history of atopic dermatitis with abscesses, re- 
current abscesses next to the various primary teeth as well as an abscess of the right popliteal area. Vigliante et al. reported a case of a 16-year-old boy with HIES and a history of recurrent dental abscesses [15]. The authors emphasized that it should be of primary concern to maxillofacial and oral surgeons that immunologic disorders quickly turn a benign dental infection into a life-threatening emergency in a very short time [15]. Furthermore, if complications arise, therapy of such patients needs to begin rapidly and should be aggressive with instant surgical incision and drainage.

Furthermore, discoloration on the lower lip in our patient was observed. As the mother reported it was related to the treatment with voriconazole (Vfend). The medicine is administered for systemic fungal infections and given for prophylactic and therapeutic purposes in patients with immunosuppression [24]. Nonetheless, it has various side effects, e.g. skin reactions which include cheilitis, discoid lupus erythematosus as well as toxic epidermal necrolysis and Stevens-Johnson syndrome. Reactions related to light exposure, i.e. environmental factor, such as phototoxicity, photosensitization, porphyria cutanea tarda and pseudoporphyria, have also been reported [24]. Furthermore, there is a presumed association between voriconazole use and cancer [25]. Consequently, mucous membrane and cutaneous symptoms of adverse effects linked to the therapy should be under constant supervision.

Literature data emphasize that oral manifestations in HIES patients include candidiasis [11, 26] as well as gingivitis and ulcerations [9]. The therapy of HIES sufferers consists of long-term prophylaxis and treatment with antibiotics, which frequently leads to mucocutaneous candidiasis, which may be also present within the oral cavity and require regular use of antifungals [27]. O'Connell et al. revealed in their study that $82 \%$ of examined HIES patients had oral candidal infection at the time of the examination [13], whereas Grimbacher et al. found chronic candidiasis of mucosal sites in $83 \%$ of individuals [3]. It is interesting that in our patient, recurrent candidiasis as well as aphthous ulcers have occurred since the birth time.

Fortunately, our patient does not display any signs of periodontal disease. Nonetheless, Tsang et al. presented a case of 5-year-old girl with suspected AR-HIES who manifested severe generalized aggressive periodontitis [20]. A polymerase chain reaction (PCR)-based microbiological examination indicated such bacteria as Porphyromonas gingivalis, Prevotella nigrescens, Tannerella forsythia, Treponema denticola, Campylobacter rectus and Eikenella corrodens in the deep periodontitis lesions. Moreover, all deciduous teeth were extracted from the patient under general anaesthesia, due to their mobility caused by alveolar bone loss [20].

A specific dental feature of AD-HIES includes failure or delay of shedding of deciduous teeth [14, 19]. Pro- longed retention of the milk teeth is caused by reduced resorption of deciduous tooth roots and prevents the appropriate eruption of the permanent successor [10]. Therefore, HIES sufferers may complain of double rows of teeth with both primary and permanent intermixed dentition [4]. Roshan et al. presented a case of a 10-yearold girl whose skull X-ray revealed retained teeth with the history of natal teeth (the upper two incisors were present at birth) as well as osteopenic lesions [16]. O'Connell et al. showed that $75 \%$ of patients above the age of 7 years reported problems with the retention of deciduous teeth including retained teeth, "double teeth" or elective extractions or exfoliation of milk teeth in adolescence and beyond [13]. Also our patient experienced retention of some deciduous molars as well as the delayed eruption of the permanent mandibular left canine and the permanent mandibular right second premolar since those teeth had to be surgically exposed in order to aid eruption.

The retention of deciduous teeth seems to be caused by a failure of these teeth to exfoliate [28]. Permanent teeth develop and erupt generally on time; however, in other cases, the failure of deciduous teeth to exfoliate may result in a delayed eruption of the permanent successor [29].

Interestingly, a clinical examination of our patient revealed a persistent lower primary right central incisor while the orthopantomogram showed a lack of the permanent successor. Kamasaki et al. presented a case of a 7-year-old girl with HIES who exhibited tooth agenesis in deciduous teeth (canines in mandible) as well as permanent dentition (lateral incisors in mandible), and deficient root resorption of deciduous mandibular central incisors which were situated in the labial position in relation to permanent successors [1]. Moreover, Domingo et al. performed research in 60 patients with HIES [17]. The authors presented a 19-year-old black female with multiple unerupted succedaneous teeth that were retained by deciduous teeth, furthermore, she had supernumerary permanent teeth (two molars and one incisor). Deepa et al. presented a case of a 18-year-old female with HIES who had retained deciduous central mandibular incisors with permanent mandibular lateral incisors congenitally missing [26]. The patient had also an infection of the gingiva with Candida albicans.

The development of dentition is regulated by many events. However, the precise molecular mechanisms are not comprehensible, though various growth factors as well as cytokines have been shown to play important roles in this process [1]. Therefore, it is suggested that an abnormal cytokine function in HIES patients affects their inflammatory response and tooth formation.

Kamasaki et al. reported dental caries in the deciduous molars of a girl with HIES [1]. The authors indicated that such a situation is due to the negligence of oral hygiene in chronically ill children, because of their poor 
physical condition. Furthermore, the frequent intake of sweetened medications, e.g. syrup, can increase the risk of dental caries. Also the personal history of our patient showed severe caries in deciduous dentition as well as its complications. She had also many permanent teeth treated because of the caries process. At present, she attends the dental surgery systematically for check-ups as well as for preventive care and dental treatment. Thus, complications of dental caries in permanent teeth may be prevented.

Since literature data showed the difficulty of diagnosis of the HIES facial characteristic in children younger than 7 years old, oral manifestations may help in the determining of the disorder [17], in particular in patients with atypical symptoms and at an earlier age.

Furthermore, patients with HIES may manifest other oral findings. Ozarmagan et al. diagnosed widespread oral papillomas in a 7-year-old girl with the syndrome [30]. Domingo et al. found out, in over $75 \%$ of HIES patients, lesions within the gingival and oral mucosa which involved the lip and buccal mucosa, the hard palate and the dorsal tongue [17]. Palatal changes mostly manifest as a midline fibrotic bridge, either linear or multilobular, and sometimes surrounded by grooves or clefts. Mucosal lesions localized within the lips and cheeks were composed of surface fissures and patches or plaques and non-rubabble keratotic striations, and some of these resemble lichenoid formations. More frequent were tongue lesions apparent as localized or widespread surface grooves. Additionally, the authors observed that all those intraoral changes were asymptomatic, occurred earlier than the typical facial manifestation as well as required no intervention. They emphasized the potential role of oral phenotypes in early diagnosis [17].

\section{Summary}

The actual development of therapeutic modalities and diagnostic tools enables health care providers to diagnose the patients very early on in their lives, prolong immunodeficient sufferers' survival, and increase lifespan as well as improve their quality of life. Since HIES is a very rare disease, it is vitally important to present such cases in the literature to inform clinicians of the significance of an early diagnosis of the disorder and appropriate therapy of general as well as dental symptoms. The cooperation between different medical professionals is necessary in providing comprehensive treatment of patients [20]. The sufferers should be under constant supervision of the specialists to avoid severe complications. It must be emphasized that different health care providers, including the paediatrician, dentist and the dermatologist, may be the first medical specialist to recognize the disorder. Thus, it is significant to broaden the specialists' knowledge about rare immunodeficiency syndromes in order to provide the sufferers with a multidisciplinary health care. Moreover, the professional should have knowledge concerning the effect of environmental factors, such as e.g. light exposure on health of patients during therapy.

HIES is of great importance to different health care providers because sufferers require special preventive and therapeutic management from early infancy in order to avoid complications which can even prove to be life-saving for such patients.

\section{Acknowledgments}

The authors would like to thank the patient and her mother for detailed information concerning general and oral health and their consent to the examination and taking pictures.

\section{Conflict of interest}

The authors declare no conflict of interest.

\section{References}

1. Kamasaki Y, Hidaka K, Nishiguchi M, Fujiwara T. Dental manifestation of a pediatric patient with hyperimmunoglobulin E syndrome: a case report. J Dent Child 2012; 79: 100-4.

2. Verma S, Wollina U. Job's syndrome - a case report. J Eur Acad Dermatol Venereol 2003; 17: 711-4.

3. Grimbacher B, Holland SM, Gallin JI, et al. Hyper-lgE syndrome with recurrent infections - an autosomal dominant multisystem disorder. N Engl J Med 1999; 340: 692-702.

4. Abhilash N, Radhakrishnan S, Arun MV, Sasidharan PK. Congenital immunodeficiency disorder. J Assoc Physicians India 2007; 55: 808-9.

5. Davis SD, Schaller J, Wedgwood RJ. Job’s syndrome. Recurrent, "cold" staphylococcal abscesses. Lancet 1966; 1: 1013-5.

6. Dewitt CA, Bishop AB, Buescher LS, Stone SP. Hyperimmunoglobulin $E$ syndrome: two cases and a review of the literature. J Am Acad Dermatol 2006; 54: 855-65.

7. Buckley RH, Wray BB, Belmaker EZ. Extreme hyperimmunoglobulinemia $E$ and undue susceptibility to infection. Pediatrics 1972; 49: 59-70.

8. Atkinson JC, O'Connell A, Aframian D. Oral manifestations of primary immunological diseases. J Am Dent Assoc 2000; 131: 345-56.

9. Deas DE, Mackey SA, McDonnell HT. Systemic disease and periodontitis: manifestations of neutrophil dysfunction. Periodontol 2000 2003; 32: 82-104.

10. Grimbacher B, Holland SM, Puck JM. Hyper-lgE syndromes. Immunol Rev 2005; 203: 244-50.

11. Hsu CT, Lin YT, Yang YH, Chiang BL. The hyperimmunoglobulin E syndrome. J Microbiol Immunol Infect 2004; 37: 121-3.

12. Muhammed K. Hyper IgE syndrome: report of two cases with moderate elevation of IgE. Indian J Dermatol Venereol Leprol 2005; 71: 112-4.

13. O'Connell AC, Puck JM, Grimbacher B, et al. Delayed eruption of permanent teeth in hyperimmunoglobulinemia $\mathrm{E}$ recurrent infection syndrome. Oral Surg Oral Med Oral Pathol Oral Radiol Endod 2000; 89: 177-85.

14. Renner ED, Puck JM, Holland SM, et al. Autosomal recessive hyperimmunoglobulin E syndrome: a distinct disease entity. J Pediatr 2004; 144: 93-9. 
15. Vigliante CE, Costello BJ, Quinn PD. Life-threatening cervicofacial infection in a child with hyperimmunoglobulin-E syndrome. J Oral Maxillofac Surg 2001; 59: 561-5.

16. Roshan AS, Janaki C, Parveen B, Gomathy N. Rare association of hyper Ig E syndrome with cervical rib and natal teeth. Indian J Dermatol 2009; 54: 372-4.

17. Domingo DL, Freeman AF, Davis J, et al. Novel intraoral phenotypes in hyperimmunoglobulin-E syndrome. Oral Dis 2008; 14: 73-81.

18. Freeman AF, Domingo DL, Holland SM. Hyper IgE (Job's) syndrome: a primary immune deficiency with oral manifestations. Oral Dis 2009; 15: 2-7.

19. Szczawinska-Poplonyk A, Kycler Z, Pietrucha B, et al. The hypeimmunoglobulin E syndrome - clinical manifestation diversity in primary immune deficiency. Orphanet J Rare Dis 2011; 6: 76.

20. Tsang P, Derkson G, Priddy R, et al. Severe periodontitis in a 5-year-old girl with hypeimmunoglobulin $E$ syndrome. Pediatr Dent 2005; 27: 68-73.

21. Smeekens SP, Huttenhower C, Riza A, et al. Skin microbiome imbalance in patients with STAT1/STAT3 defects impairs innate host defense responses. I Innate Immun 2014; 6: 253-62.

22. Tonsi A. Hyper-lgE syndrome. A study involving 30 children from Makkah - Saudi Arabia. Eur J Pediat Dermatol 2004; 14: 209-14.

23. Pherwani AV, Madnani NA. Hyperimmunoglobulin E syndrome. Indian Pediatr 2001; 38: 1029-34.

24. Vöhringer S, Schrum J, Ott H, Höger PH. Severe phototoxicity associated with long-term voriconazole treatment. J Deutch DG 2011; 9: 274-6.

25. Goyal RK. Voriconazole-associated phototoxic dermatoses and skin cancer. Expert Rev Anti Infect Ther 2015; 13: 1537-46.

26. Deepa D, Kumar KV, Joshi CS, et al. Fungal infection of gingiva in a patient with hyperimmunoglobulin-E (Job's) syndrome. J Indian Soc Periodontol 2012; 16: 256-60.

27. McAuliffe NJ, Hunter ML, Kau CH, et al. The dental management of a patient with hyperimmunoglobulinemia $\mathrm{E}$ syndrome: a case report. Int J Paediatr Dent 2005; 15: 127-30.

28. Freeman AF, Holland SM. The hyper-lgE syndromes. Immunol Allergy Clin North Am 2008; 28: 277-91.

29. Szczawinska-Poplonyk A, Gerreth K, Breborowicz A, Borysewicz-Lewicka M. Oral manifestations of primary immune deficiencies in children. Oral Surg Oral Med Oral Pathol Oral Radiol Endod 2009; 108: e9-20.

30. Ozarmagan G, Yazganoglu KD, Agacfidan A. Hyper-Ig E syndrome with widespread premalign oral papillomas treated with Interferon a2b. Acta Derm Venereol 2005; 85: 433-5. 